\title{
Comparison of contrast-enhanced T1-weighted imaging using DANTE-SPACE, PETRA, and MPRAGE: a clinical evaluation of brain tumors at 3 Tesla
}

\author{
Qing Fu ${ }^{1,2} \wedge$, Qi-Guang Cheng ${ }^{1,2}$, Xiang-Chuang Kong ${ }^{1,2}$, Ding-Xi Liu ${ }^{1,2} \wedge$, Yi-Hao Guo ${ }^{3}$,

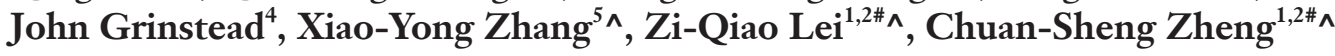 \\ ${ }^{1}$ Department of Radiology, Union Hospital, Tongji Medical College, Huazhong University of Science and Technology, Wuhan, China; ${ }^{2}$ Hubei \\ Province Key Laboratory of Molecular Imaging, Wuhan, China; ${ }^{3}$ MR Collaboration, Siemens Healthcare Ltd., Guangzhou, China; ${ }^{4}$ Siemens \\ Medical Solutions USA, Inc., Portland, OR, USA; ${ }^{5}$ Clinical Science, Philips Healthcare, Chengdu, China
}

Contributions: (I) Conception and design: Q Fu, ZQ Lei, CS Zheng; (II) Administrative support: ZQ Lei, CS Zheng; (III) Provision of study materials or patients: QG Cheng, XC Kong, DX Liu; (IV) Collection and assembly of data: Q Fu, QG Cheng, XC Kong, DX Liu; (V) Data analysis and interpretation: Q Fu, YH Guo, J Grinstead, XY Zhang; (VI) Manuscript writing: All authors; (VII) Final approval of manuscript: All authors.

\#These authors contributed equally to this work.

Correspondence to: Prof. Chuan-Sheng Zheng. Department of Radiology, Union Hospital, Tongji Medical College, Huazhong University of Science and Technology, 1277 Jiefang Avenue, Wuhan 430022, China. Email: hqzcsxh@sina.com.

Background: We aimed to compare the performance of three contrast-enhanced T1-weighted threedimensional (3D) magnetic resonance (MR) sequences to detect brain tumors at 3 Tesla. The three sequences were: (I) delay alternating with nutation for tailored excitation sampling perfection with applicationoptimized contrasts using different flip angle evolution (DANTE-SPACE), (II) pointwise encoding time reduction with radial acquisition (PETRA), and (III) magnetization-prepared rapid acquisition with gradient echo (MPRAGE).

Methods: This study involved 77 consecutive patients, including 34 patients with known primary brain tumors and 43 patients suspected of intracranial metastases. All patients underwent each of the three sequences with comparable spatial resolution and acquisition time post-injection. Signal-to-noise ratios (SNRs) for gray matter (GM) and white matter (WM), contrast-to-noise ratios (CNRs) for lesion/GM, lesion/WM, and GM/WM were quantitatively compared. Two radiologists determined the total number of enhancing lesions by consensus. Intraclass correlation coefficients (ICCs) between the two radiologists for metastases presence, qualitative ratings for image quality, and acoustic noise level of each sequence were assessed.

Results: Among the three sequences, SNRs and CNRs between lesions and surrounding parenchyma were highest using DANTE-SPACE, but $\mathrm{CNR}_{W M / G M}$ was the lowest with DANTE-SPACE. SNRs for PETRA images were significantly higher than those for MPRAGE $(\mathrm{P}<0.001)$. CNRs between lesions and surrounding parenchyma were similar for PETRA and MPRAGE $(\mathrm{P}>0.05)$. Significantly more brain metastases were detected with DANTE-SPACE ( $n=94)$ compared with MPRAGE ( $n=71)$ and PETRA ( $n=72)$. The ICCs were 0.964 for MPRAGE, 0.975 for PETRA, and 0.973 for DANTE-SPACE. Qualitative scores for lesion imaging using DANTE-SPACE were significantly higher than those obtained with PETRA and MPRAGE ( $\mathrm{P}=0.002$ and $\mathrm{P}=0.004$, respectively). The acoustic noise level for PETRA (64.45 dB) was significantly lower

^ ORCID: Qing Fu, 0000-0003-0102-0276; Qi-Guang Cheng, 0000-0001-9488-131X; Xiang-Chuang Kong, 0000-0001-5740-0425; DingXi Liu, 0000-0001-8886-1419; Yi-Hao Guo, 0000-0002-3294-4351; Xiao-Yong Zhang, 0000-0001-7667-8676; Zi-Qiao Lei, 0000-00023103-4188; Chuan-Sheng Zheng, 0000-0002-2435-1417. 
than that for MPRAGE $(78.27 \mathrm{~dB}, \mathrm{P}<0.01)$ and DANTE-SPACE $(80.18 \mathrm{~dB}, \mathrm{P}<0.01)$.

Conclusions: PETRA achieves comparable detection of brain tumors with MPRAGE and is preferred for depicting osseous metastases and meningeal enhancement. DANTE-SPACE with blood vessel suppression showed improved detection of cerebral metastases compared with MPRAGE and PETRA, which could be helpful for the differential diagnosis of tumors.

Keywords: Magnetization-prepared rapid acquisition with gradient echo (MPRAGE); delay alternating with nutation for tailored excitation sampling perfection with application-optimized contrasts using different flip angle evolution (DANTE-SPACE); pointwise encoding time reduction with radial acquisition (PETRA); brain tumor; intracranial metastasis

Submitted Jan 27, 2021. Accepted for publication Jul 13, 2021.

doi: 10.21037/qims-21-107

View this article at: https://dx.doi.org/10.21037/qims-21-107

\section{Introduction}

Primary and metastatic brain tumors are life-threatening due to their infiltrative and invasive nature and are associated with high patient morbidity and mortality (1). For some brain tumors, the identification of genetic features influences the diagnosis, treatment, and prognosis (2). In clinical settings, the optimal therapeutic strategies for brain tumors generally depend on the number, size, location, and grading of the tumors; therefore, early and accurate diagnosis is of utmost importance (3).

Magnetic resonance imaging (MRI) has been widely accepted as the most sensitive imaging modality. It is preferred for assessing the lesion type, size, location, extent, enhancement characteristics, and malignancy of brain tumors (4) by depicting the anatomical and functional features of tumors with high tissue contrast. The administration of gadolinium-based contrast material assists with early and accurate differential diagnosis and tumor grading by depicting margins and enhancing morphological information within lesions. This information is essential for informing clinical decisions about surgical resection, radiotherapy, or chemotherapy, including delineating appropriate target volumes and managing follow-up regarding disease recurrence and treatment outcomes $(5,6)$.

Contrast-enhanced T1-weighted imaging (T1WI) MRI, which is widely used in the standard clinical assessment of brain tumors, can be conducted using either threedimensional (3D) or two-dimensional (2D) magnetic resonance (MR) sequences. With thinner-section thicknesses in 3D datasets for reconstructing arbitrary orientation slice images of the $3 \mathrm{D}$ volumetric acquisition datasets, 3D MR sequences are recommended for cerebral tumor detection (7). They have been demonstrated to be superior to $2 \mathrm{D}$ sequences to detect and visualize small brain metastases with reduced partial volume effect and fewer blood flow artifacts (8-11).

Magnetization-prepared rapid acquisition with gradient echo (MPRAGE) is a 3D sequence used for neuroimaging that provides excellent tissue contrast between gray matter (GW) and white matter (WM), has high spatial resolution with isotropic imaging (12), and is less sensitive to pulsation artifacts (13). Thus, it is commonly used for contrastenhancement evaluation of intracranial tumors (14). However, contrast-enhanced T1-weighted MPRAGE shows hyperintensity in both arterial and venous vessels, similar to small contrast-enhanced focal lesions; this hinders the accurate detection of lesions, especially for small metastatic tumors located in regions near the cerebral cortex or sulci $(15-17)$. This disadvantage limits its use for the diagnosis of small metastatic tumors. Moreover, MPRAGE has a limited role in depicting lesions with slight enhancement concentration (13). In a recent investigation (18), MPRAGE demonstrated inferior visualization of brain tumor enhancement compared to sampling perfection with application-optimized contrasts using different flip angle evolution (SPACE) and volumetric interpolated brain examination (VIBE).

Pointwise encoding time reduction with radial acquisition (PETRA), a quiet 3D sequence alternative to MPRAGE, combines half-projection radial acquisition in outer k-space and single pointwise measurement on a Cartesian trajectory in center k-space, and it has an ultra-short echo time (TE) of $0.07 \mathrm{~ms}$ (19). The PETRA sequence applies an inversion recovery pulse to yield T1WI and has shown performance comparable with the MPRAGE sequence in pediatric 
brain imaging (20) and postcontrast intracranial tumors with a much lower acoustic noise level compared with conventional MPRAGE (21). However, after administering the contrast material, vessels are enhanced to hyperintensity in PETRA; this may cause difficulty in differentiating focal lesions from small vessels when using this sequence.

Black-blood techniques can suppress signal intensity in arterial and venous vessels by nulling the inflowing blood (22) through various strategies, including delay alternating with nutation for tailored excitation (DANTE) (23), spatial presaturation (24), double inversion recovery (25), and motion-sensitizing magnetization preparation (26). The DANTE preparation module institutes nonselective low flip angle pulse trains interspersed with gradient pulses with short interpulse spacing. This enables static tissues to preserve most of their transverse coherence while flowing spins are substantially attenuated with short interpulse repeat times combined with gradient fields along the flow direction during the DANTE pulse trains, achieving substantial attenuation of moving spins while retaining static signals (23). According to the basic rationale of the DANTE preparation module, the signal suppression of DANTE is insensitive to velocities, which provides excellent blood signal suppression for arteries and veins with slow blood flow. Combining the blood signal suppression of DANTE with SPACE, 3D DANTE-SPACE has been used clinically for vessel wall imaging of carotid and intracranial arteries $(23,27)$; diagnosing deep venous thrombosis (28); and has been shown to detect small metastatic lesions effectively (23).

To the best of the authors' knowledge, no previous studies have compared the three 3D T1WI sequences DANTE-SPACE, PETRA, and MPRAGE to diagnose brain tumors. This study aimed to compare the diagnostic performance and image quality of these three $3 \mathrm{D}$ contrastenhanced T1WI sequences to detect metastatic and primary brain tumors at 3 Tesla $(\mathrm{T})$. By comparing the different properties of the three sequences, we hypothesized the following: (I) due to the black-blood intrinsic characteristic of DANTE-SPACE, this sequence might provide superior tumor conspicuity and may be more useful for differentiation of perivascular tumors compared with MPRAGE and PETRA; and (II) PETRA might provide comparable image quality with MPRAGE and have superior performance in depicting cranial bones and meningeal enhancement compared to the other two sequences, which could be attributed to its special ultra-short TE.

\section{Methods}

\section{Patient population}

The study was conducted following the Declaration of Helsinki (as revised in 2013). The study was approved by the Medical Ethics Committee of Huazhong University of Science and Technology, and written informed consent was taken from all individual participants.

Between February 2019 and August 2020, a total of 77 consecutive patients (43 males and 34 females; mean age, 51.6 years; age range, $20-73$ years) were enrolled in the study. Of these patients, 43 (25 males and 18 females; mean age, 55.3 years; age range, $32-73$ years) with known malignancy underwent cranial contrast-enhanced MR scanning for evaluation of intracranial metastases. The diagnosis of the primary malignant tumors was lung cancer $(\mathrm{n}=37)$ and breast cancer $(\mathrm{n}=6)$, and each patient with metastases had at least 1 follow-up MRI after radiation therapy with or without chemotherapy. The remaining 34 patients ( 18 males and 16 females; mean age, 47.0 years; age range, $20-71$ years) had identified primary brain tumors, including meningioma $(n=9)$, glioma $(n=22)$, hemangiopericytoma $(n=2)$, and lymphoma $(\mathrm{n}=1)$. All enrolled patients underwent contrastenhanced MR examinations according to clinical standards.

\section{MRI protocol}

All MR examinations were performed on a $3 \mathrm{~T}$ MR scanner system (Magnetom Skyra, Siemens Healthineers, Erlangen, Germany) equipped with $45 \mathrm{mT} / \mathrm{m}$ achievable gradient strength and $200 \mathrm{~T} / \mathrm{m} / \mathrm{s}$ maximum slew rate, using a 20-channel head-neck coil. Foam pads were placed at the bilateral sides of the head within the head coil to fix the patient's position and help decrease the acoustic noise heard by patients. All patients were instructed to remain still during the MR image acquisition. Pre-contrast sequences were scanned in 2D mode, including T1-weighted, T2weighted, fluid-suppressed T2-weighted, and diffusionweighted images. The contrast-enhanced MR sequence used in our department was T1-weighted MPRAGE, and contrast-enhanced DANTE-SPACE and PETRA T1weighted sequences were added in this study. The three contrast-enhanced T1-weighted MR sequences were used with the same spatial resolution and comparable acquisition time in 3D mode. One minute after administration of gadobutrol (Gadovist; Bayer Schering Pharma, Berlin, Germany) at a standard dose $(0.1 \mathrm{~mL} / \mathrm{kg}$ body weight $)$ at 
Table $1 \mathrm{MR}$ parameters for the three T1WI sequences

\begin{tabular}{|c|c|c|c|}
\hline Parameters & MPRAGE & PETRA & DANTE-SPACE \\
\hline 3D or 2D mode & $3 D$ & $3 \mathrm{D}$ & $3 D$ \\
\hline Coverage & Whole brain & Whole brain & Whole brain \\
\hline Orientation & Sagittal & Axial & Sagittal \\
\hline TE (ms) & 2.29 & 0.07 & 4.6 \\
\hline $\mathrm{FOV}\left(\mathrm{mm}^{2}\right)$ & 240 & 300 & 280 \\
\hline Bandwidth (Hz/pixel) & 200 & 401 & 524 \\
\hline Number of slices & 176 & 320 & 192 \\
\hline Flip angle $\left(^{\circ}\right)$ & 8 & 6 & Variable \\
\hline Voxel size $\left(\mathrm{mm}^{3}\right)$ & $0.94 \times 0.94 \times 0.94$ & $0.94 \times 0.94 \times 0.94$ & $0.94 \times 0.94 \times 0.94$ \\
\hline TA & $5 \min 21 \mathrm{~s}$ & $5 \min 57 \mathrm{~s}$ & $5 \min 33 s$ \\
\hline
\end{tabular}

MR, magnetic resonance; T1WI, T1-weighted imaging; MPRAGE, magnetization-prepared rapid acquisition with gradient echo; PETRA, pointwise encoding time reduction with radial acquisition; DANTE-SPACE, delay alternating with nutation for tailored excitation sampling perfection with application-optimized contrasts using different flip angle evolution; GRE, gradient recalled echo; TSE, turbo spin echo; 3D or 2D mode, three-dimensional or two-dimensional mode; TR, repetition time; TE, echo time; FOV, field of view; GRAPPA, generalized auto calibrating partially parallel acquisition; TA, acquisition time.

a rate of $1.5 \mathrm{~mL} / \mathrm{s}$, the three sequences were scanned in a random order to avoid timing bias which might be caused by different concentrations of gadolinium-based contrast material over time after injection. The detailed parameters for each of the sequences are shown in Table 1.

\section{MR image analysis}

For the 43 patients with suspected brain metastases, the diagnostic criteria for brain metastases were determined as follows (29): (I) enhancing structures with a diameter of more than $0.5 \mathrm{~mm}$ that appeared as a lesion instead of being an artifact or normal enhanced anatomical structure; and (II) contrast-enhancing lesions that were visualized in only one of the contrast-enhanced T1-weighted sequences.

The images were evaluated in two sessions. In the first session, two experienced radiologists (with 10 and 15 years of experience in neuroimaging) independently assessed the sequences on a picture archiving and communication system (PACS). The two reviewers read and analyzed only one type of sequence each time, and a 1-week interval was used to avoid memory bias for the image review of the three different 3D sequences. The reading order was arranged randomly. The reviewers were permitted to make multiplanar reconstructions using MR Workstation 3D software (Siemens). The reviewers were not blinded to the clinical purposes of each enrolled patient for MRI, but other patient information was removed from PACS to ensure the blind-reading mode. For each reviewer, the presence, absence, and number of enhancing lesions were determined. In the second session, 1 month after the initial analyses, the two radiologists together read and compared the images generated for each sequence to determine the presence or absence of an enhancing lesion. The time allotted for the two radiologists to review the images was not limited. A decision was made to determine the final result by consensus as a reference standard in the case of disagreement, and the reasons for false-positive and falsenegative lesions were also analyzed by comparing their initial findings with the reference standard. The final number and maximum diameter of lesions were recorded. Intraclass correlation coefficients (ICCs) between the two radiologists for diagnosing the presence of metastatic lesions were analyzed for interobserver agreement. 


\section{Subjective evaluation for all the enrolled patients}

In the second session of image quality analyses, the two radiologists together scored the overall image quality of the three sequences using a 4-point scale (4: excellent, no artifacts; 3: good, minor artifacts; 2: adequate, major artifacts; and 1: insufficient for diagnosis) to evaluate the suitability of MR images for adequate diagnosis (30).

Additionally, the images of enhancing lesions were also evaluated by consensus using a 4-point scale (4: excellent; 3: good; 2: fair; and 1: poor) based on the following diagnostic information: (I) lesion border delineation, (II) definition of lesion extent, (III) visualization of internal lesion morphology, and (IV) lesion contrast enhancement compared with the surrounding normal tissues (31).

\section{Objective evaluation for all the enrolled patients}

Region-of-interest (ROI) analyses were performed for all three sequences. Signal intensities (SI) of GM, WM, and lesions were measured for calculating signal-to-noise ratios (SNRs) and contrast-to-noise ratios (CNRs) for all three sequences. SNRs for GW and WM, and CNRs for lesion/ WM, lesion/GM, and WM/GM were calculated using the following formulas (30):

$$
\begin{aligned}
& \mathrm{SNR}_{\mathrm{WM}}=\mathrm{SI}_{\mathrm{WM}} / \mathrm{SD}_{\mathrm{WM}} \\
& \mathrm{SNR}_{\mathrm{GM}}=\mathrm{SI}_{\mathrm{GM}} / \mathrm{SD}_{\mathrm{GM}} \\
& \mathrm{CNR}_{\text {lesion } / \mathrm{WM}}=\left(\mathrm{SI}_{\text {lesion }}-\mathrm{SI}_{\mathrm{WM}}\right) / \mathrm{SD}_{\mathrm{WM}} \\
& \mathrm{CNR}_{\text {lesion } / \mathrm{GM}}=\left(\mathrm{SI}_{\text {lesion }}-\mathrm{SI}_{\mathrm{GM}}\right) / \mathrm{SD}_{\mathrm{GM}} \\
& \mathrm{CNR}_{\mathrm{WM} / \mathrm{GM}}=\left(\mathrm{SI}_{\mathrm{WM}}-\mathrm{SI}_{\mathrm{GM}}\right) / \mathrm{SD}_{\mathrm{WM}}
\end{aligned}
$$

SD represents the standard deviation of the signal in the respective ROI. To evaluate $\mathrm{CNR}_{\text {lesion/WM }}$ and $\mathrm{CNR}_{\text {lesion/GM }}$, lesions with homogeneous solid enhancement, clear margins, and a diameter of $5 \mathrm{~mm}$ or larger were selected. Lesions less than $5 \mathrm{~mm}$ in diameter were excluded from the quantitative assessment because of the difficulty in placing a ROI within those lesions. The circular ROIs of enhancing lesions were placed in the center of the lesions without involving the lesion margins. The ROIs of WM were placed at the genu of the corpus callosum, and the ROIs of GM with a size of $20 \mathrm{~mm}^{2}$ were placed at the head of the normal caudate nucleus (29). All measurements of WM and GM were made, avoiding vessels, artifacts, and any contrastenhancing lesions.

\section{Measurement of acoustic noise level}

The acoustic levels of the three sequences were recorded using a decibel meter placed laterally 1 meter from the lateral panel of the MR scanner core. For each patient, real-time noise levels were measured and recorded every 20 seconds and repeated 15 times during the scanning of each sequence. The average noise levels for each sequence were then calculated for further comparisons.

\section{Statistical analysis}

All statistical analyses were performed using SPSS (IBM Corp., Armonk, NY, USA). The variables were presented as mean \pm SD. Kruskal-Wallis one-way ANOVA and Bonferroni correction were used for statistical analysis. ICC estimates between the two reviewers were calculated based on absolute agreement; a two-way random model was used for reliability analysis (poor reliability: $<0.5$; moderate reliability, 0.5-0.75; good reliability: 0.75-0.9; excellent reliability: $>0.9) . \mathrm{P}<0.05$ was considered statistically significant.

\section{Results}

\section{Patient population, lesion number, and diameters}

One minute after contrast material injection, the three sequences were scanned in random order. Details of the scan orders for the enrolled patients are listed in Table 2. Two female patients (52 and 45 years, both with identified lung cancers) were excluded from the study due to difficulty counting the numerous metastatic lesions. As illustrated in Figure 1, more enhancing lesions were observed using DANTE-SPACE than with MPRAGE and PETRA. A significantly greater number of brain metastases were detected with DANTE-SPACE (94 lesions) than with MPRAGE (71 lesions) and PETRA (72 lesions). Figure 2 shows normal representative images obtained using MPRAGE, PETRA, and DANTE-SPACE. Arterial and venous vessels were enhanced to hyperintensity with both MPRAGE and PETRA. For DANTE-SPACE images, the signal suppression was complete for arterial vessels but was incomplete for some venous vessels (Figure 2).

The maximum diameters of enhancing metastatic lesions were seen using PETRA $(7.46 \pm 7.70 \mathrm{~mm})$, MPRAGE $(7.50 \pm 7.72 \mathrm{~mm})$, and DANTE-SPACE $(7.51 \pm 7.71 \mathrm{~mm})$ showed no significant statistical difference $(\mathrm{P}=0.993)$. 
Table 2 Details of scan orders of post-contrast T1WI sequences for all patients

\begin{tabular}{|c|c|c|}
\hline Scan orders & Patient number & Interval time between contrast injection and the beginning of each sequence acquisition \\
\hline$A-B-C$ & 12 & $1 \min -6 \min 21 s-12 \min 18 s$ \\
\hline$A-C-B$ & 13 & $1 \min -6 \min 21 \mathrm{~s}-11 \min 54 \mathrm{~s}$ \\
\hline$B-C-A$ & 14 & $1 \min -6 \min 57 \mathrm{~s}-12 \min 30 \mathrm{~s}$ \\
\hline$B-A-C$ & 12 & $1 \min -6 \min 57 s-12 \min 18 s$ \\
\hline $\mathrm{C}-\mathrm{B}-\mathrm{A}$ & 12 & $1 \min -6 \min 33 s-12 \min 30 \mathrm{~s}$ \\
\hline
\end{tabular}

A represents post-contrast MPRAGE sequence; $B$ represents post-contrast PETRA sequence; $C$ represents post-contrast DANTE-SPACE sequence. T1WI, T1-weighted imaging; DANTE-SPACE, delay alternating with nutation for tailored excitation sampling perfection with application-optimized contrasts using different flip angle evolution; MPRAGE, magnetization-prepared rapid acquisition with gradient echo; PETRA, pointwise encoding time reduction with radial acquisition.
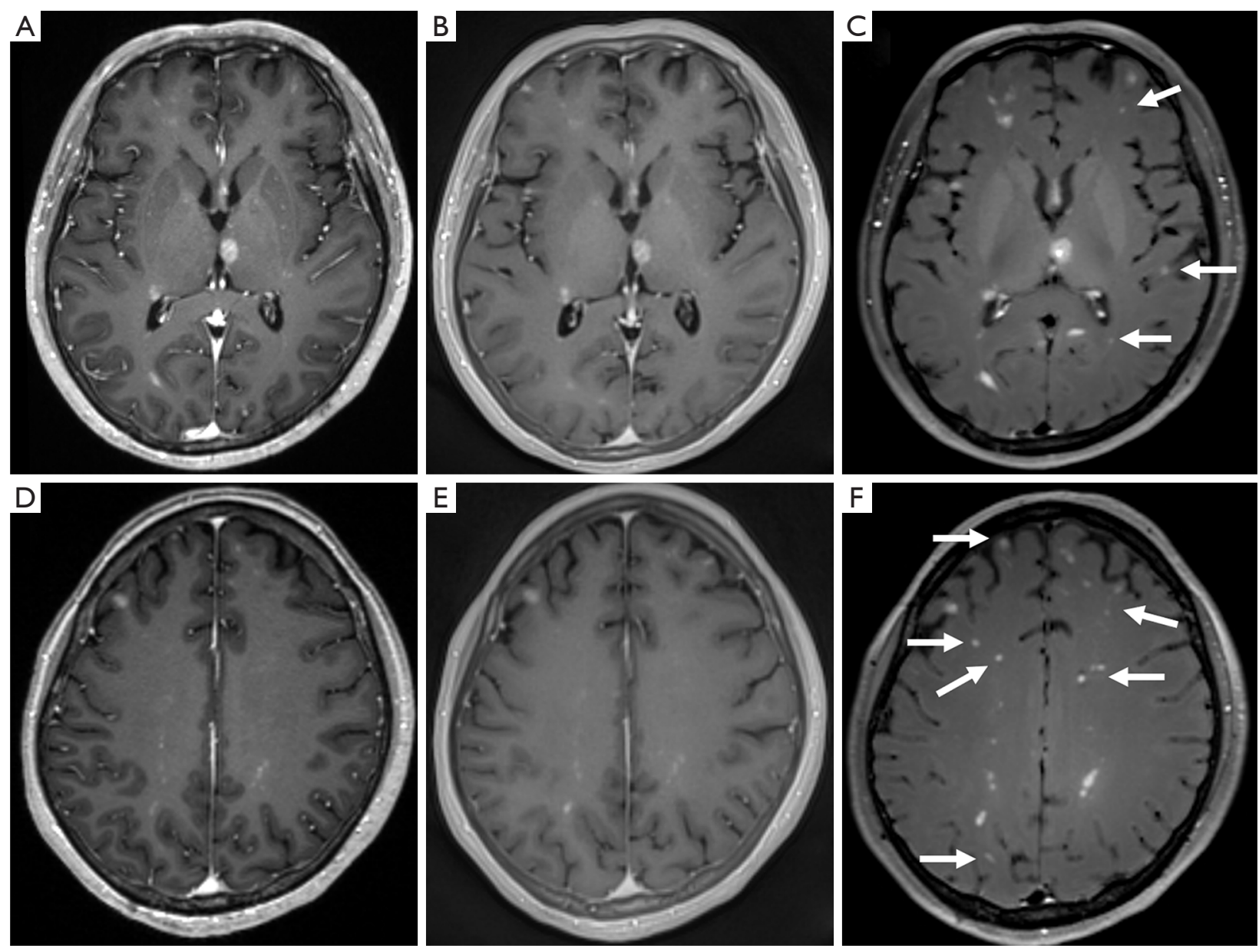

Figure 1 Images for a 52-year-old female patient with numerous enhancing lesions using MPRAGE (A,D), PETRA (B,E), and DANTESPACE (C,F). The enhancing lesions obtained with DANTE-SPACE (C,F) show better-defined margins and contrast between lesions and surrounding parenchyma compared with those obtained with MPRAGE (A,D) and PETRA (B,E). More enhancing lesions were detected with DANTE-SPACE [arrows in (C,F)] than with MPRAGE and PETRA. MPRAGE, magnetization-prepared rapid acquisition with gradient echo; PETRA, pointwise encoding time reduction with radial acquisition; DANTE-SPACE, delay alternating with nutation for tailored excitation sampling perfection with application-optimized contrasts using different flip angle evolution. 

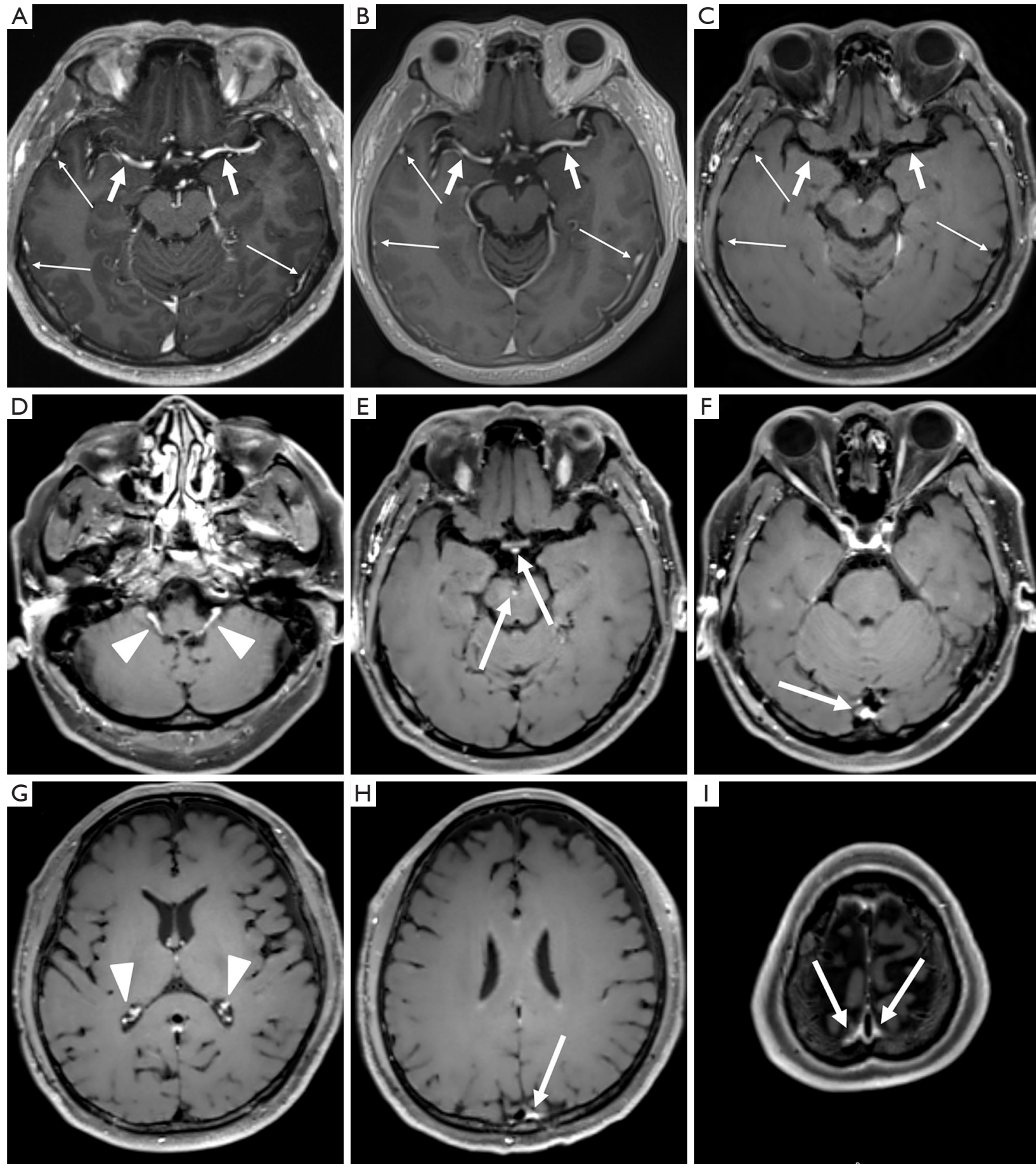

Figure 2 Representative images obtained using MPRAGE (A), PETRA (B), and DANTE-SPACE (C) in a normal brain, and representative images of DANTE-SPACE at different levels from skull base to head vertex (D,E,F,G,H,I). (A,B) Arterial vessels (short arrows) and small vessels located in brain surface (long arrows) showed signal hyperintensity; (C) the vessels were completely suppressed (short arrows). (E,F,H,I) suppression of some venous vessels was incomplete (long arrows); (D,G) suppression of contrast-enhanced choroid plexus was incomplete (arrowheads). MPRAGE, magnetization-prepared rapid acquisition with gradient echo; PETRA, pointwise encoding time reduction with radial acquisition; DANTE-SPACE, delay alternating with nutation for tailored excitation sampling perfection with application-optimized contrasts using different flip angle evolution. 

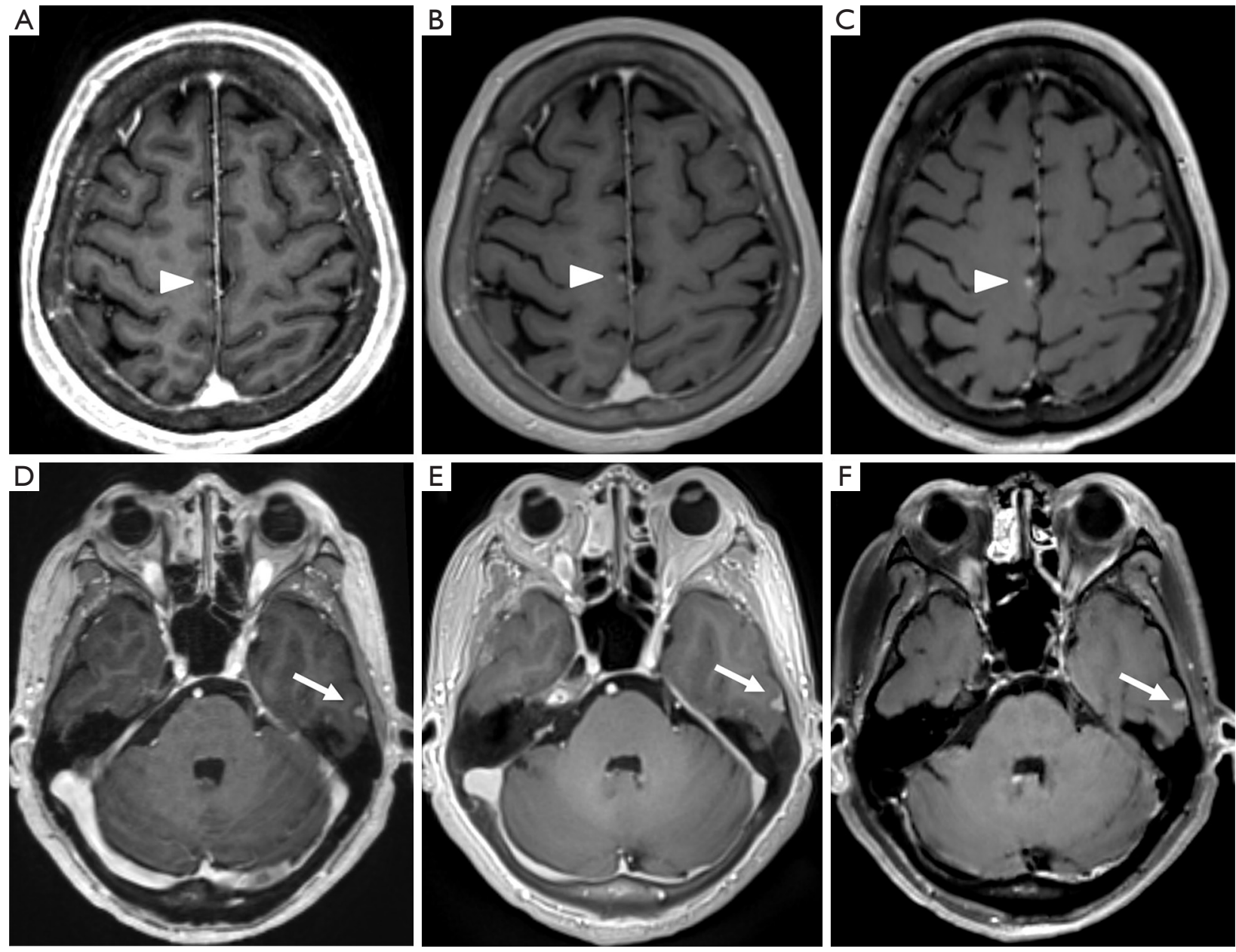

Figure 3 Representative images of MPRAGE (A,D), PETRA (B,E), and DANTE-SPACE (C,F) of a 57-year-old male patient with cerebral metastases of small-cell lung cancer. In the axial images (A,B,C), the contrast between WM and GM decreased from A to C. A wellenhanced lesion near the cerebral falx in the right frontal lobe can be seen using all three sequences (arrowheads); the lesion appeared to be more visible and slightly larger with DANTE-SPACE than in the MPRAGE and PETRA images. This patient also had a small meningioma in the left temporal lobe (D,E,F); the homogeneously enhanced lesion was displayed clearly with well-defined margins using all three sequences (arrows). MPRAGE, magnetization-prepared rapid acquisition with gradient echo; PETRA, pointwise encoding time reduction with radial acquisition; DANTE-SPACE, delay alternating with nutation for tailored excitation sampling perfection with applicationoptimized contrasts using different flip angle evolution; WM, white matter; GM, gray matter.

The ICCs between the two radiologists for the presence of metastatic lesions was 0.964 for MPRAGE, 0.975 for PETRA, and 0.973 for DANTE-SPACE.

By comparing the respective initial findings of the two radiologists with the consensus results for the presence or absence of enhancing lesions, the number of false-negative results for metastatic lesions missing in the initial diagnosis for MPRAGE, PETRA, and DANTE-SPACE, were 19, 19 , and 12 , respectively. These lesions were mostly in the vicinity of the cerebral falx or the peripheral and surface areas of the brain with diameters of $0.5-3 \mathrm{~mm}$ (Figure 3). The number of false positives for metastatic lesions was two for DANTE-SPACE and one for PETRA.

In the identification of primary tumors, there was no significant difference among MPRAGE, PETRA, and DANTE-SPACE and the number $(n=22, n=22, n=22$, respectively; $\mathrm{P}=1.000$ ) or maximum diameter of primary brain tumors $(3.45 \pm 1.87,3.49 \pm 1.85,3.49 \pm 1.85 \mathrm{~cm}$, respectively; $\mathrm{P}=0.998)$. However, the homogeneity of signal intensity within some tumors differed among the 
three sequences (Figure 4), with DANTE-SPACE showing heterogeneous signal intensities while MPRAGE and PETRA showed homogenous imaging.

The diagnosis of intracranial and skull metastases in a 71-year-old breast cancer patient (Figure 5), which was validated by single-photon emission computed tomography (SPECT), had been missed in the initial diagnosis. Additionally, a 57-year-old male patient had a suspicious venous malformation in the diploe of the skull that was detected with all sequences. The conterminous vein flowed back into the left internal jugular vein, and the signal intensity of this small vein was enhanced in both MPRAGE and PETRA but suppressed in DANTE-SPACE.

\section{Subjective evaluation}

All the MR images met the standard of diagnostic image quality, and no significant differences were found in the overall image quality score among the three sequences $(\mathrm{P}=0.415)$. The image scores for lesion depiction for each sequence are listed as follows: PETRA, 3.76 \pm 0.43 ; MPRAGE, 3.75 \pm 0.44 ; DANTE-SPACE, 3.96 \pm 0.20 . There was no significant difference between the scores obtained using PETRA and MPRAGE $(\mathrm{P}=0.999)$; however, the score obtained using DANTE-SPACE was significantly higher than those obtained with PETRA and MPRAGE ( $\mathrm{P}=0.002$, $\mathrm{P}=0.004$, respectively).

\section{Objective evaluation}

The mean values of objective parameters for each sequence were listed in Table 3, including $\mathrm{SNR}_{\mathrm{WM}}, \mathrm{SNR}_{\mathrm{GM}}$, $\mathrm{CNR}_{\text {lesion/GM }}, \mathrm{CNR}_{\text {lesion/WM }}$, and $\mathrm{CNR}_{\mathrm{WM} / \mathrm{GM}}$.

$\mathrm{SNR}_{W M}$ and $\mathrm{SNR}_{\mathrm{GM}}$ values obtained using DANTESPACE were significantly higher than those obtained with PETRA ( $\mathrm{P}<0.001, \mathrm{P}<0.001$, respectively), while the same values using PETRA were significantly higher than those obtained with MPRAGE $(\mathrm{P}<0.001, \mathrm{P}<0.001$, respectively).

There was a strong contrast between enhancing lesions and parenchyma tissue when the DANTE-SPACE sequence was used. The $\mathrm{CNR}_{\text {lesion/GM }}$ and $\mathrm{CNR}_{\text {lesion/WM }}$ values obtained using DANTE-SPACE were significantly higher than those obtained with MPRAGE and PETRA $(\mathrm{P}<0.001, \mathrm{P}<0.001$, respectively), while the values obtained with MPRAGE were similar to that of PETRA ( $\mathrm{P}=0.268$, $\mathrm{P}=0.999$, respectively).

The tissue contrast between GM and WM was highest in MPRAGE images and lowest in DANTE-SPACE images. The $\mathrm{CNR}_{\mathrm{WM} / \mathrm{GM}}$ value obtained using MPRAGE was significantly higher than that obtained using PETRA $(\mathrm{P}=0.008)$, while the $\mathrm{CNR}_{\mathrm{WM} / \mathrm{GM}}$ value obtained using PETRA was significantly higher than that obtained with DANTE-SPACE $(\mathrm{P}<0.001)$.

\section{Acoustic noise level}

The acoustic noise level of the PETRA sequence $(64.45 \pm 1.52 \mathrm{~dB})$ was significantly lower than that of MPRAGE $(78.27 \pm 2.18 \mathrm{~dB}, \mathrm{P}<0.01)$. The noise level of the MPRAGE sequence was significantly lower than that of DANTE-SPACE $(80.18 \pm 1.10 \mathrm{~dB}, \mathrm{P}<0.01)$.

\section{Discussion}

We compared the diagnostic performance of three 3D postcontrast T1-weighted sequences (MPRAGE, PETRA, and DANTE-SPACE) for imaging brain tumors in the present study. The results suggest that DANTE-SPACE provided better CNR between lesions and surrounding tissues and was superior for visualizing enhancing lesions, especially for small metastatic lesions. The PETRA sequence provided significantly quieter acoustic noise levels and comparable diagnostic value when compared with MPRAGE.

Compared to MPRAGE, PETRA may be able to achieve similar image quality for brain tumors. In the current study, the SNR of GM and WM in PETRA was significantly higher than that of MPRAGE, while no significant difference between PETRA and MPRAGE was detected in the CNR of lesions and parenchyma. This result is consistent with a previous study (21) which reported that PETRA was an alternative to MPRAGE for contrastenhanced intracranial tumors. Flow-related artifacts and susceptibility artifacts have previously been reported to be reduced or absent using PETRA (32,33), which has been studied in the imaging of paranasal sinuses and inferior temporal areas (21).

In the current study, the ability of PETRA to visualize detailed structures of some short T2 components, such as cranial and laminar bones and meningeal enhancement, was superior to that of MPRAGE and DANTE-SPACE. This may have been because the TE of PETRA (TE $=0.07 \mathrm{~ms}$ ) is much shorter than that of MPRAGE (TE $=2.29 \mathrm{~ms}$ ) and DANTE-SPACE ( $\mathrm{TE}=4.6 \mathrm{~ms})$. In a comparison study between PETRA and MPRAGE for brain tumors by Ida et al. (21), the cranial bones and dura enhancement were observed more clearly on PETRA than MPRAGE, but 

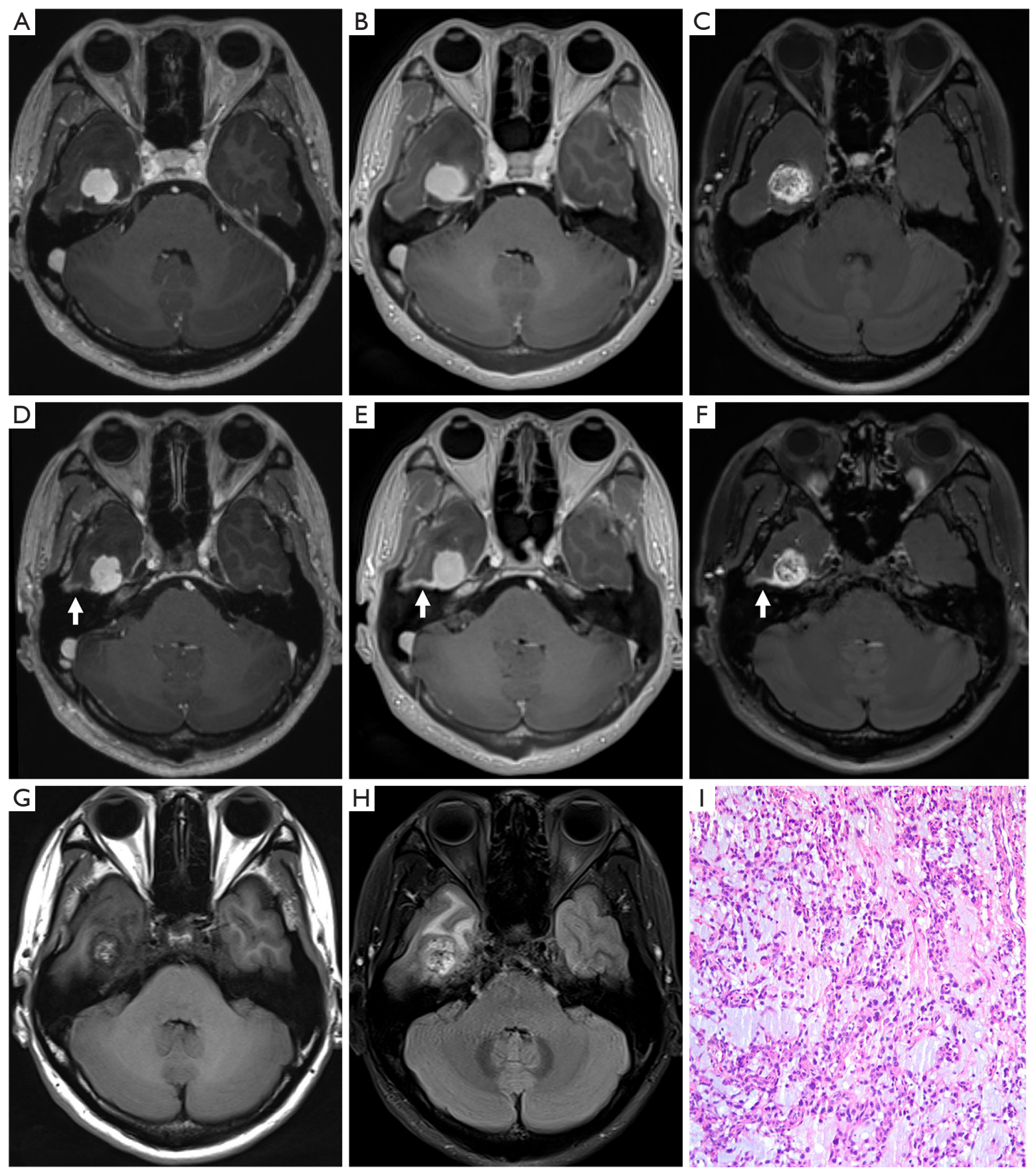

Figure 4 Post-contrast MPRAGE (A,D), PETRA (B,E), and DANTE-SPACE (C,F) images and pre-contrast T1WI and T2WI (G,H) images of a myopericytoma in the right temporal lobe of a 37-year-old female patient. The signal intensity within this tumor was relatively homogeneous in MPRAGE (A,D) and PETRA (B,E), but was heterogeneous with DANTE-SPACE (C,F). The enhancing effect of adjacent meninges (arrows) was more continuous and more obvious in PETRA (E) compared with MPRAGE (D) and DANTE-SPACE (F). The internal tumor imaging seen in DANTE-SPACE (C,F) was in line with the heterogeneous imaging of pre-contrast images $(\mathrm{G}, \mathrm{H})$; this tumor was identified as a myopericytoma by surgical pathology (I, hematoxylin-eosin stain, $\times 100$ ). MPRAGE, magnetization-prepared rapid acquisition with gradient echo; PETRA, pointwise encoding time reduction with radial acquisition; DANTE-SPACE, delay alternating with nutation for tailored excitation sampling perfection with application-optimized contrasts using different flip angle evolution; T1WI, T1weighted imaging; T2WI, T2-weighted imaging. 

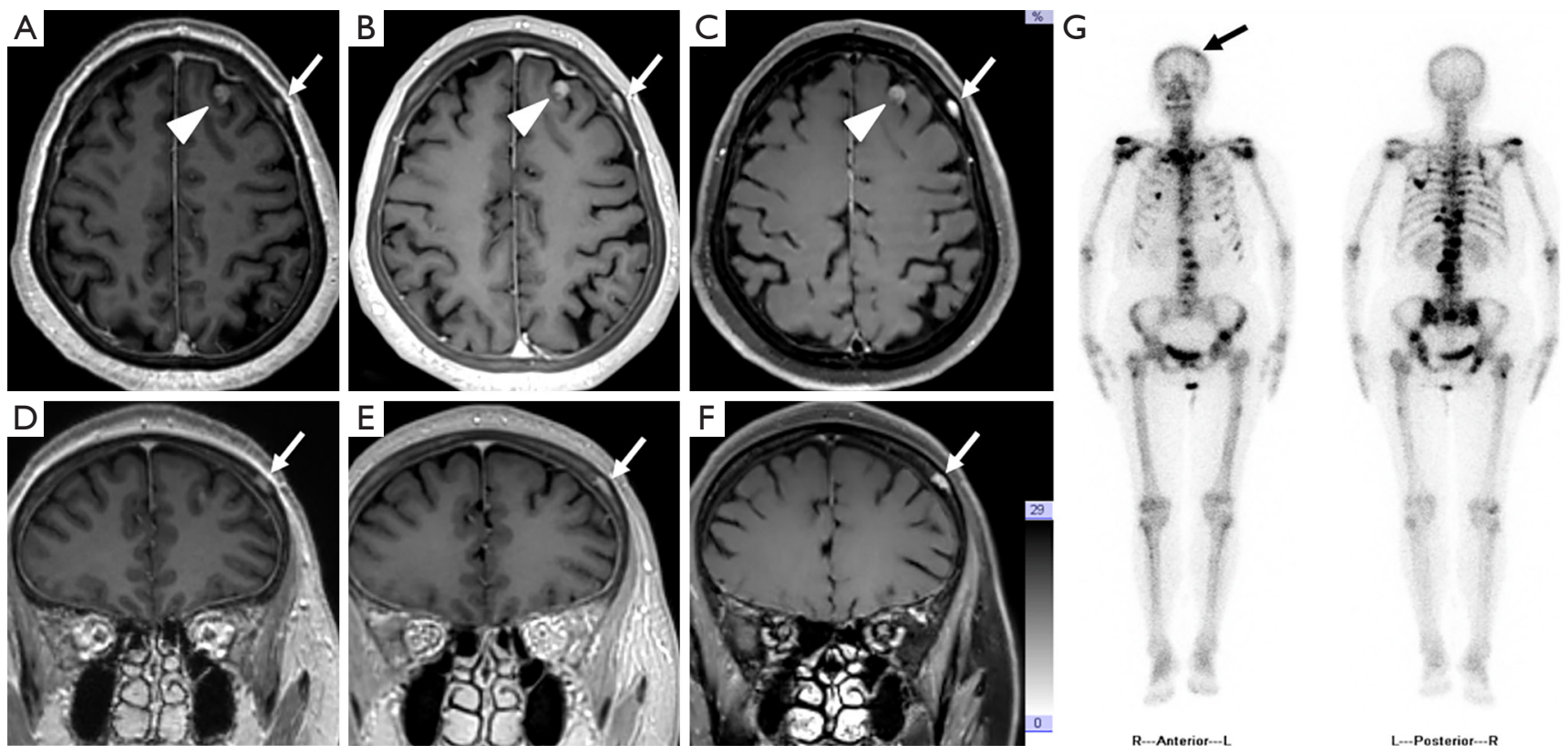

Figure 5 Post-contrast axial (A,B,C) and coronal (D,E,F) T1WI of a 71-year-old female patient with identified breast cancer obtained using MPRAGE (A,D), PETRA (B,E), and DANTE-SPACE (C,F). The enhancing lesion in the intracranial parenchyma [arrowheads in $(\mathrm{A}, \mathrm{B}, \mathrm{C})]$ and skull [white arrows in $(\mathrm{A}, \mathrm{B}, \mathrm{C}, \mathrm{D}, \mathrm{E}, \mathrm{F})]$ were visualized using all three sequences. The detailed structures of the skull diploe were depicted more clearly by PETRA than by MPRAGE and DANTE-SPACE. Anterior and posterior images of whole-body scan by SPECT (G) showed hyperactive metabolism in the spine, ribs, and left frontal-parietal skull (black arrows), suggesting multiple bone metastases. T1WI, T1-weighted imaging; MPRAGE, magnetization-prepared rapid acquisition with gradient echo; PETRA, pointwise encoding time reduction with radial acquisition; DANTE-SPACE, delay alternating with nutation for tailored excitation sampling perfection with application-optimized contrasts using different flip angle evolution; SPECT, single-photon emission computed tomography.

Table 3 Quantitative analyses of the three sequences for imaging intracranial tumors greater than $5 \mathrm{~mm}$ in diameter

\begin{tabular}{lccc}
\hline Parameters & MPRAGE & PETRA & DANTE-SPACE \\
\hline SNR $_{\mathrm{WM}}$ & $33.04 \pm 12.16$ & $49.00 \pm 32.71$ & $67.13 \pm 26.87$ \\
$\mathrm{SNR}_{\mathrm{GM}}$ & $26.15 \pm 9.88$ & $34.61 \pm 9.83$ & $63.65 \pm 23.02$ \\
$\mathrm{CNR}_{\text {lesion/GM }}$ & $25.93 \pm 20.86$ & $19.52 \pm 13.97$ & $61.80 \pm 115.69$ \\
$\mathrm{CNR}_{\text {lesion/WM }}$ & $19.63 \pm 24.84$ & $15.85 \pm 13.82$ & $89.11 \pm 194.83$ \\
$\mathrm{CNR}_{\mathrm{WM} / \mathrm{GM}}$ & $8.18 \pm 21.50$ & $7.53 \pm 2.82$ & $-4.56 \pm 4.51^{\mathrm{a}}$ \\
\hline
\end{tabular}

a, signal intensities of WM were lower than those of GM in DANTE-SPACE, so this value is shown as a negative number. MPRAGE, magnetization-prepared rapid acquisition with gradient echo; PETRA, pointwise encoding time reduction with radial acquisition; DANTE-SPACE, delay alternating with nutation for tailored excitation sampling perfection with application-optimized contrasts using different flip angle evolution; SNR, signal-to-noise ratio; CNR, contrast-to-noise ratio; WM, white matter; GM, gray matter.

the performance of PETRA for bone metastases was not compared. We also found that PETRA could supplement new options for depicting osseous metastases and provide more uniform enhancement of the involved meninges than DANTE-SPACE and MPRAGE, which is clearly illustrated in Figures 4,5. Moreover, our study showed that the CNR between GM and WM was significantly higher using PETRA compared with DANTE-SPACE. This may make it difficult to localize certain lesions accurately using DANTE-SPACE. 
Additionally, PETRA provided a lower acoustic noise level than either MPRAGE or DANTE-SPACE. Almost all MRI sequences cause considerable acoustic noise during MR scanning, which is generally produced by fast switching and vibration of the magnetic field gradient coils. Previous reports have shown PETRA to be a quieter sequence. Because the gradient remains constant throughout the entire repetition with very slight changes at the end of each repetition without being ramped down, the result is very slight vibrations from the gradient coil switching (21). This could provide a more comfortable environment for patients, decreasing their inner anxiety and increasing the success rate of MR examination, which may be particularly helpful for children and patients with mental disorders (34). MPRAGE and DANTE-SPACE each have a higher acoustic noise level than PETRA, which may be related to the vibrations of gradient fields and magnetization-prepared or DANTE-prepared pulses, respectively.

Our study showed that the DANTE-SPACE sequence detected more enhancing lesions than PETRA and MPRAGE, especially for small metastases. This may be explained by the higher SNR of the spin-echo nature of SPACE and the blood vessel suppression of combining the DANTE module with SPACE. This finding of the present study is consistent with a prior study (29), in which DANTE-SPACE significantly improved detection of brain metastases over MPRAGE.

We also found that, compared with MPRAGE and PETRA, DANTE-SPACE might be more useful for the differential diagnosis of perivascular tumors, and to the best of our knowledge, this finding has not yet been reported. As shown in Figure 4, the signal intensity within this tumor was homogeneous when using either MPRAGE or PETRA, while it was extremely heterogeneous when displayed in DANTE-SPACE with several blackened small vessels. While this tumor was initially suspected of glioma, it was later determined to be a myopericytoma by postoperative histopathology. Myopericytoma is a rare tumor mostly found in subcutaneous and superficial soft tissues and is characterized by numerous thin-walled blood vessels (35). Due to the enhancement of blood vessels and solid components within these tumors, the tissue contrast between them may be relatively difficult to differentiate, resulting in a homogeneous enhancement on MPRAGE and PETRA. However, due to the blood signal suppression of DANTE-SPACE, blood vessels within this tumor are blackened to hypo-intensity, thus revealing the nonhomogeneity within this tumor, which is in line with the precontrast T1WI and $\mathrm{T} 2$-weighted imaging (T2WI) images as well as the surgical pathology. This finding suggests that DANTE-SPACE might be more accurate for differential diagnosis of perivascular tumors compared with MPRAGE and PETRA.

Signal suppression of arterial vessels was observed to be excellent in DANTE-SPACE but was incomplete for some slow venous flows, such as the peripheral venous sinuses or small veins on the brain surface. Although the DANTE module is used for signal suppression of moving spins while largely preserving the signals from static spins, the combination of DANTE with SPACE can significantly increase arterial and venous blood suppression compared with the SPACE sequence alone (27). This may result in misdiagnosis (15). In a report by Kim et al. (29), DANTESPACE produced more false-positive cases $(n=18)$ than MPRAGE $(\mathrm{n}=10)$ due to incomplete vessel suppression $(n=15)$ and flow-related artifacts $(n=3)$.

In contrast, our results showed only 1-2 false-positive metastatic lesions in DANTE-SPACE and PETRA, while 12-19 false-negative metastatic lesions were detected. This may have been due to the following reasons.

First, the two radiologists in the current study were familiar with the imaging features of DANTE-SPACE, PETRA, and MPRAGE, and thus the residual blood signals in DANTE-SPACE were generally recognized. Although contrast-enhanced small vessels may mimic the imaging of small metastatic lesions, they may be differentiated by using multiplanar reconstruction techniques in combination with the detailed location, with small vessels continuing across multiple adjacent slices. Therefore, the number of falsepositive lesions in the present study was very low.

Second, by using a combination of three sequences as reference standards in the current study, we found that 19 lesions were missed in the initial diagnoses for both MPRAGE alone and PETRA alone, and 12 for DANTESPACE alone. Those false-negative lesions could be found by comparing the corresponding locations by multiplanar reconstructions but were misinterpreted as normal contrast-enhanced vessels on the brain surface. Although the radiologists were highly experienced in differentiating lesions from enhancing anatomical structures, it was challenging to identify every small metastatic lesion by examining the axial, sagittal, coronal, and oblique planes, especially when they were located near small vessels. Moreover, there were 170-320 slices for each sequence due to the 0.94 slice thickness used with the $3 \mathrm{D}$ model. The number of slices would have gone up to 510-940 if axial, 
coronal, and sagittal images were all used for observation.

While previous similar studies have focused on PETRA, DANTE-SPACE, and MPRAGE $(21,29)$, the additional findings of the current study could help clinicians and radiologists to choose the best option for different clinical scenarios: (I) DANTE-SPACE would be recommended for demonstrating small brain metastases with the best lesion conspicuity; moreover, it is more useful for differentiating perivascular tumors than MPRAGE and PETRA. (II) The quieter PETRA sequence is more suitable for patients who cannot tolerate loud acoustic noise, and it is recommended if cranial bone and/or meninges are suspected to be involved in brain tumors as it has comparable image qualities with MPRAGE.

There were several limitations to this study. First, the number of enrolled patients was relatively small. Further, as it typically requires about 5-6 minutes to scan the three sequences used in the study, there are likely to be motion artifacts. Although all patients in the study were cooperative during the MR scanning, and PETRA may be immune to slight movement due to the radial acquisition part of the sequence, there is still a need to decrease the scan time of the $3 \mathrm{D}$ volumetric acquisitions to improve workflow and reduce the possibility of patient motion. Therefore, accelerated techniques, such as the compressed sensing technique (36), could be considered in future studies.

Second, PETRA scanned in the axial plane due to its combination feature, while MPRAGE and DANTESPACE images were acquired with both sagittal planes. However, although the section orientation differed among the three $3 \mathrm{D}$ sequences, the same isotropic voxel size of $0.94 \mathrm{~mm}^{3}$ was applied for all three sequences with similar acquisition times.

Third, the diagnosis of brain metastases lacked pathological confirmation in our study. However, instead of surgical resection, stereotactic radiosurgery and wholebrain radiation therapy are commonly used with multiple brain metastases $(37,38)$. Therefore, it was reasonable for the clinical diagnostic results to be determined by contrastenhanced T1-weighed imaging.

Fourth, in the interest of remaining conservative, enhancing lesions that were considered too small $(<0.5 \mathrm{~mm})$ were excluded from the study. Therefore, the true number of enhancing metastatic brain lesions may be more than the number reported here. Nonetheless, we consider the results of the comparison among the three T1WI sequences to be credible as we have used clinical criteria for brain metastases that are widely accepted.
Fifth, various primary tumors were included in the current study. Future studies should focus on vascularrelated tumors to validate the usefulness of differential diagnoses among the three sequences, especially for DANTE-SPACE.

Sixth, the three T1WI sequences evaluated in the current study were scanned in a random order to avoid the timing bias that might occur due to the time-dependency of brain tumor enhancement; however, we believe that this did not affect our accuracy results. The completion of the last sequence occurred nearly 20 minutes post-injection, and it has been previously found that excellent brain tumor visualization was obtained between $8.5-38.5$ minutes postinjection (39).

Lastly, the 1-minute delay from contrast injection to the first post-contrast image acquisition may have led to potential bias in the current study.

\section{Conclusions}

PETRA imaging may achieve comparable detection for brain tumors with lower acoustic noise levels compared with MPRAGE, and it is preferred for depicting osseous metastases and meningeal enhancement. Compared with MPRAGE and PETRA, DANTE-SPACE imaging with blood vessel suppression demonstrated improved detection of small cerebral metastases and increased CNRs between lesions and surrounding parenchyma, and therefore could be effective for determining the differential diagnosis of tumors.

\section{Acknowledgments}

Funding: None.

\section{Footnote}

Conflicts of Interest: All authors have completed the ICMJE uniform disclosure form (available at https://dx.doi. org/10.21037/qims-21-107). The authors have no conflicts of interest to declare.

Ethical Statement: The authors are accountable for all aspects of the work in ensuring that questions related to the accuracy or integrity of any part of the work are appropriately investigated and resolved. The study was conducted in accordance with the Declaration of Helsinki (as revised in 2013). The study was approved by the Medical 
Ethics Committee of Huazhong University of Science and Technology, and written informed consent was taken from all individual participants.

Open Access Statement: This is an Open Access article distributed in accordance with the Creative Commons Attribution-NonCommercial-NoDerivs 4.0 International License (CC BY-NC-ND 4.0), which permits the noncommercial replication and distribution of the article with the strict proviso that no changes or edits are made and the original work is properly cited (including links to both the formal publication through the relevant DOI and the license). See: https://creativecommons.org/licenses/by-nc-nd/4.0/.

\section{References}

1. Moffat BA, Chenevert TL, Lawrence TS, Meyer CR, Johnson TD, Dong Q, Tsien C, Mukherji S, Quint DJ, Gebarski SS, Robertson PL, Junck LR, Rehemtulla A, Ross BD. Functional diffusion map: a noninvasive MRI biomarker for early stratification of clinical brain tumor response. Proc Natl Acad Sci U S A 2005;102:5524-9.

2. Farrell CJ, Plotkin SR. Genetic causes of brain tumors: neurofibromatosis, tuberous sclerosis, von Hippel-Lindau, and other syndromes. Neurol Clin 2007;25:925-46, viii.

3. Schellinger PD, Meinck HM, Thron A. Diagnostic accuracy of MRI compared to CCT in patients with brain metastases. J Neurooncol 1999;44:275-81.

4. Brant-Zawadzki M, Norman D, Newton TH, Kelly WM, Kjos B, Mills CM, Dillon W, Sobel D, Crooks LE. Magnetic resonance of the brain: the optimal screening technique. Radiology 1984;152:71-7.

5. Essig M, Weber MA, von Tengg-Kobligk H, Knopp MV, Yuh WT, Giesel FL. Contrast-enhanced magnetic resonance imaging of central nervous system tumors: agents, mechanisms, and applications. Top Magn Reson Imaging 2006;17:89-106.

6. Warnke PC, Kreth FW, Ostertag CB. Early postoperative magnetic resonance imaging after resection of malignant glioma: objective evaluation of residual tumor and its influence on regrowth and prognosis. Neurosurgery 1995;36:872-4.

7. Wetzel SG, Johnson G, Tan AG, Cha S, Knopp EA, Lee VS, Thomasson D, Rofsky NM. Three-dimensional, T1-weighted gradient-echo imaging of the brain with a volumetric interpolated examination. AJNR Am J Neuroradiol 2002;23:995-1002.

8. Schwindt W, Kugel H, Bachmann R, Kloska S, Allkemper
T, Maintz D, Pfleiderer B, Tombach B, Heindel W. Magnetic resonance imaging protocols for examination of the neurocranium at 3 T. Eur Radiol 2003;13:2170-9.

9. Kakeda S, Korogi Y, Hiai Y, Ohnari N, Moriya J, Kamada K, Hanamiya M, Sato T, Kitajima M. Detection of brain metastasis at 3T: comparison among SE, IR-FSE and 3D-GRE sequences. Eur Radiol 2007;17:2345-51.

10. Takeda T, Takeda A, Nagaoka T, Kunieda E, Takemasa K, Watanabe M, Hatou T, Oguro S, Katayama M. Gadolinium-enhanced three-dimensional magnetizationprepared rapid gradient-echo (3D MP-RAGE) imaging is superior to spin-echo imaging in delineating brain metastases. Acta Radiol 2008;49:1167-73.

11. Chen W, Wang L, Zhu W, Xia L, Qi J, Feng D, Luo $\mathrm{X}$. Multicontrast single-slab 3D MRI to detect cerebral metastasis. AJR Am J Roentgenol 2012;198:27-32.

12. Mugler JP 3rd, Brookeman JR. Three-dimensional magnetization-prepared rapid gradient-echo imaging (3D MP RAGE). Magn Reson Med 1990;15:152-7.

13. Blüml S, Schad LR, Scharf J, Wenz F, Knopp MV, Lorenz WJ. A comparison of magnetization prepared 3D gradientecho (MP-RAGE) sequences for imaging of intracranial lesions. Magn Reson Imaging 1996;14:329-35.

14. Ellingson BM, Bendszus M, Boxerman J, Barboriak D, Erickson BJ, Smits M, et al. Consensus recommendations for a standardized Brain Tumor Imaging Protocol in clinical trials. Neuro Oncol 2015;17:1188-98.

15. Nagao E, Yoshiura T, Hiwatashi A, Obara M, Yamashita K, Kamano H, Takayama Y, Kobayashi K, Honda H. 3D turbo spin-echo sequence with motion-sensitized driven-equilibrium preparation for detection of brain metastases on 3T MR imaging. AJNR Am J Neuroradiol 2011;32:664-70.

16. Park J, Kim J, Yoo E, Lee H, Chang JH, Kim EY. Detection of small metastatic brain tumors: comparison of 3D contrast-enhanced whole-brain black-blood imaging and MP-RAGE imaging. Invest Radiol 2012;47:136-41.

17. Kato Y, Higano S, Tamura H, Mugikura S, Umetsu A, Murata T, Takahashi S. Usefulness of contrast-enhanced T1-weighted sampling perfection with applicationoptimized contrasts by using different flip angle evolutions in detection of small brain metastasis at 3T MR imaging: comparison with magnetization-prepared rapid acquisition of gradient echo imaging. AJNR Am J Neuroradiol 2009;30:923-9.

18. Danieli L, Riccitelli GC, Distefano D, Prodi E, Ventura E, Cianfoni A, Kaelin-Lang A, Reinert M, Pravatà E. Brain Tumor-Enhancement Visualization and Morphometric 
Assessment: A Comparison of MPRAGE, SPACE, and VIBE MRI Techniques. AJNR Am J Neuroradiol 2019;40:1140-8.

19. Grodzki DM, Jakob PM, Heismann B. Ultrashort echo time imaging using pointwise encoding time reduction with radial acquisition (PETRA). Magn Reson Med 2012;67:510-8.

20. Aida N, Niwa T, Fujii Y, Nozawa K, Enokizono M, Murata K, Obata T. Quiet T1-Weighted Pointwise Encoding Time Reduction with Radial Acquisition for Assessing Myelination in the Pediatric Brain. AJNR Am J Neuroradiol 2016;37:1528-34.

21. Ida M, Wakayama T, Nielsen ML, Abe T, Grodzki DM. Quiet T1-weighted imaging using PETRA: initial clinical evaluation in intracranial tumor patients. J Magn Reson Imaging 2015;41:447-53.

22. Kawaji K, Nguyen TD, Zou Z, Reig B, Winchester PA, Shih A, Spincemaille P, Prince MR, Wang Y. Threedimensional flow-independent balanced steady-state free precession vessel wall MRI of the popliteal artery: preliminary experience and comparison with flowdependent black-blood techniques. J Magn Reson Imaging 2011;34:696-701.

23. Li L, Miller KL, Jezzard P. DANTE-prepared pulse trains: a novel approach to motion-sensitized and motionsuppressed quantitative magnetic resonance imaging. Magn Reson Med 2012;68:1423-38.

24. Ehman RL, Felmlee JP. Flow artifact reduction in MRI: a review of the roles of gradient moment nulling and spatial presaturation. Magn Reson Med 1990;14:293-307.

25. Chien D, Goldmann A, Edelman RR. High-speed black blood imaging of vessel stenosis in the presence of pulsatile flow. J Magn Reson Imaging 1992;2:437-41.

26. Wang J, Yarnykh VL, Hatsukami T, Chu B, Balu N, Yuan C. Improved suppression of plaque-mimicking artifacts in black-blood carotid atherosclerosis imaging using a multislice motion-sensitized driven-equilibrium (MSDE) turbo spin-echo (TSE) sequence. Magn Reson Med 2007;58:973-81.

27. Xie Y, Yang Q, Xie G, Pang J, Fan Z, Li D. Improved black-blood imaging using DANTE-SPACE for simultaneous carotid and intracranial vessel wall evaluation. Magn Reson Med 2016;75:2286-94.

28. Zhuang G, Tang C, He X, Liang J, He Z, Ye Y, Deng W, Liu D, Chen H. DANTE-SPACE: a new technical tool for DVT on 1.5T MRI. Int J Cardiovasc Imaging 2019;35:2231-7.

29. Kim D, Heo YJ, Jeong HW, Baek JW, Han JY, Lee JY, Jin
SC, Baek HJ. Usefulness of the Delay Alternating with Nutation for Tailored Excitation Pulse with T1-Weighted Sampling Perfection with Application-Optimized Contrasts Using Different Flip Angle Evolution in the Detection of Cerebral Metastases: Comparison with MPRAGE Imaging. AJNR Am J Neuroradiol 2019;40:1469-75.

30. Kammer NN, Coppenrath E, Treitl KM, Kooijman H, Dietrich O, Saam T. Comparison of contrast-enhanced modified T1-weighted 3D TSE black-blood and 3D MPRAGE sequences for the detection of cerebral metastases and brain tumours. Eur Radiol 2016;26:1818-25.

31. Rowley HA, Scialfa G, Gao PY, Maldjian JA, Hassell D, Kuhn MJ, Wippold FJ 2nd, Gallucci M, Bowen BC, Schmalfuss IM, Ruscalleda J, Bastianello S, Colosimo C. Contrast-enhanced MR imaging of brain lesions: a large-scale intraindividual crossover comparison of gadobenate dimeglumine versus gadodiamide. AJNR Am J Neuroradiol 2008;29:1684-91.

32. Fu Q, Zhang XY, Deng XB, Liu DX. Clinical evaluation of subtracted pointwise encoding time reduction with radial acquisition-based magnetic resonance angiography compared to 3D time-of-flight magnetic resonance angiography for improved flow dephasing at 3 Tesla. Magn Reson Imaging 2020;73:104-10.

33. van den Hauwe L, Parizel PM, Van Goethem JW, De Schepper AM. Clinical usefulness of contrast-enhanced MP-RAGE of the brain. Neuroradiology 1996;38 Suppl 1:S14-9.

34. Fu Q, Liu DX, Zhang XY, Deng XB, Zheng CS. Pointwise encoding time reduction with radial acquisition in subtraction-based magnetic resonance angiography to assess saccular unruptured intracranial aneurysms at 3 Tesla. Neuroradiology 2021;63:189-99.

35. Stout AP, Murray MR. Hemangiopericytoma: a vascular tumor featuring zimmermann's pericytes. Ann Surg 1942;116:26-33.

36. Jia S, Zhang L, Ren L, Qi Y, Ly J, Zhang N, Li Y, Liu $\mathrm{X}$, Zheng H, Liang D, Chung YC. Joint intracranial and carotid vessel wall imaging in 5 minutes using compressed sensing accelerated DANTE-SPACE. Eur Radiol 2020;30:119-27.

37. Linskey ME, Andrews DW, Asher AL, Burri SH, Kondziolka D, Robinson PD, Ammirati M, Cobbs CS, Gaspar LE, Loeffler JS, McDermott M, Mehta MP, Mikkelsen T, Olson JJ, Paleologos NA, Patchell RA, Ryken TC, Kalkanis SN. The role of stereotactic radiosurgery in the management of patients with newly diagnosed brain 
metastases: a systematic review and evidence-based clinical practice guideline. J Neurooncol 2010;96:45-68.

38. Chang WS, Kim HY, Chang JW, Park YG, Chang JH. Analysis of radiosurgical results in patients with brain metastases according to the number of brain lesions: is stereotactic radiosurgery effective for multiple brain

Cite this article as: Fu Q, Cheng QG, Kong XC, Liu DX, Guo YH, Grinstead J, Zhang XY, Lei ZQ, Zheng CS. Comparison of contrast-enhanced T1-weighted imaging using DANTE-SPACE, PETRA, and MPRAGE: a clinical evaluation of brain tumors at 3 Tesla. Quant Imaging Med Surg 2022;12(1):592-607. doi: 10.21037/qims-21-107 metastases? J Neurosurg 2010;113 Suppl:73-8.

39. Schörner W, Laniado M, Niendorf HP, Schubert C, Felix $\mathrm{R}$. Time-dependent changes in image contrast in brain tumors after gadolinium-DTPA. AJNR Am J Neuroradiol 1986;7:1013-20. 